University of Wollongong

Research Online

Faculty of Engineering and Information

Faculty of Engineering and Information

Sciences - Papers: Part A

Sciences

2013

On the weighting of the mean-absolute-deviation cost minimization model

S Wang

National University of Singapore, shuaian@uow.edu.au

Q Meng

National University of Singapore

Z Liu

National University of Singapore

Follow this and additional works at: https://ro.uow.edu.au/eispapers

Part of the Engineering Commons, and the Science and Technology Studies Commons

Research Online is the open access institutional repository for the University of Wollongong. For further information contact the UOW Library: research-pubs@uow.edu.au 


\title{
On the weighting of the mean-absolute-deviation cost minimization model
}

\begin{abstract}
The mean-absolute-deviation cost minimization model, which aims to minimize sum of the mean value and the absolute deviation (AD) of the total cost multiplied by a given non-negative weighting, is one of a number of typical robust optimization models. This paper first uses a straightforward example to show that the solution obtained by this model with some weightings is not actually an optimal decision. This example also illustrates that the mean-absolute-deviation cost minimization model cannot be regarded as the conventional weighted transformation of the relevant multiobjective minimization model aiming to simultaneously minimize the mean value and AD. This paper further proves that the optimal solution obtained by the mean-absolute-deviation cost minimization model with the weighting not exceeding 0.5 will not be absolutely dominated by any other solution. This tight upper bound provides a useful guideline for practical applications.
\end{abstract}

\section{Keywords}

weighting, cost, deviation, mean, minimization, absolute, model

Disciplines

Engineering | Science and Technology Studies

\section{Publication Details}

Wang, S., Meng, Q. \& Liu, Z. (2013). On the weighting of the mean-absolute-deviation cost minimization model. Journal of the Operational Research Society, 64 622-628. 


\title{
On the weighting of the mean-absolute-deviation cost minimization model
}

\author{
Shuaian Wang, Qiang Meng * Zhiyuan Liu \\ Department of Civil and Environmental Engineering, National University of Singapore,
} Singapore 117576

\begin{abstract}
The mean-absolute-deviation cost minimization model, which aims to minimize sum of the mean value and the absolute deviation of the total cost multiplied by a given non-negative weighting, is one of the typical robust optimization models. This paper first uses a straightforward example to show that the solution obtained by this model with some weightings is actual not an optimal decision. This example also illustrates that the meanabsolute-deviation cost minimization model cannot be regarded as the conventional weighted transformation of the relevant multiobjective minimization model aiming to simultaneously minimize the mean value and absolute deviation. This paper further proves that the optimal solution obtained by the mean-absolute-deviation cost minimization model with the weighting not exceeding 0.5 will not be absolutely dominated by any other solution. This tight upper bound provides a useful guideline for practical applications.
\end{abstract}

Key Words: Optimization; Multiobjective; Mean-absolute-deviation

\footnotetext{
${ }^{*}$ Corresponding author

Tel.: +65 65165494

Fax: +65 67791635

E-mail address: ceemq@nus.edu.sg (Q. Meng)
} 


\section{Introduction}

Robust optimization is a field of optimization theory that deals with optimization problems where robustness is sought against uncertainty and/or variability in the value of a parameter or a solution of the problem. One line of research on robust optimization seeks to optimize the worst-case scenario (e.g., Ben-Tal et al., 2009). The other line of research aims to control the variability of the objective function in different scenarios. Mulvey et al. (1995) contributed to the second line of research by proposing the following mean-variance cost minimization model to cope with the uncertain parameters involved in the robust optimization model building:

$$
\min _{x \in X} C_{\mathrm{MV}}(x)=\sum_{s \in S} p_{s} \xi_{s}(x)+\lambda \sum_{s \in S} p_{s}\left(\xi_{s}(x)-\sum_{s^{\prime} \in S} p_{s^{\prime}} \xi_{s^{\prime}}(x)\right)^{2}
$$

This model is a weighted sum of the expected value and variance of the random cost, where $X \subseteq \mathfrak{R}^{n}$ is the set of feasible solutions; $S$ is the set of all possible scenarios (or realizations) of the uncertain parameters; $\xi_{s}(x)$ is a deterministic cost incurred when the decision variable takes a particular value $x \in X$ for a specific scenario or realization of the uncertain parameters, $s \in S ; p_{s}$ is the occurrence probability of scenario $s \in S$ and $\lambda$ is a given nonnegative weighting to balance the expected value of the random cost, expressed by the first term on the right-hand side of Eq. (1), and its variance with the weighting $\lambda$ shown by the second term on the right-hand side of Eq. (1). These occurrence probabilities should fulfill the fundamental condition:

$$
\sum_{s \in S} p_{s}=1
$$


For the ease of exposition, this paper assumes that any solution $x \in X$ is feasible to all the scenarios of the uncertain parameters. In other words, this paper only focuses on the solution robustness issue.

It should be pointed out that taking the variance as one component of risk measure has its origin in Markowitz (1952). The variance term in the above mean-variance cost minimization model possesses a quadratic form resulting in more computing burden when solving the model. As a consequence, researchers have proposed several approaches to linearize the risk measure (see, Mansini et al., 2003). The absolute deviation (AD) is a frequently used approach for measuring the variability of the random cost since it is linear programming solvable. Absolute deviation is defined as

$$
A D(x)=\sum_{s \in S} p_{s}\left|\xi_{s}(x)-\sum_{s^{\prime} \in S} p_{s^{\prime}} \xi_{s^{\prime}}(x)\right|
$$

As a variation of the mean-variance cost minimization model, the mean-absolute-deviation cost minimization model can thus be formulated below:

$$
\min _{x \in X} C_{\mathrm{MAD}}(x)=\sum_{s \in S} p_{s} \xi_{s}(x)+\lambda \sum_{s \in S} p_{s}\left|\xi_{s}(x)-\sum_{s^{\prime} \in S} p_{s^{\prime}} \xi_{s^{\prime}}(x)\right|
$$

The mean-absolute-deviation cost minimization model was first proposed by Konno and Yamazaki (1991) and later used in logistics analysis by Yu and Li (2000).

In the mean-absolute-deviation cost minimization model (4), the weighting $\lambda$ plays a role to control the variability of the resulting cost of the solution. In other words, the variability of the optimal solution, measure by $\sum_{s \in S} p_{s}\left|\xi_{s}(x)-\sum_{s^{\prime} \in S} p_{s^{\prime}} \xi_{s^{\prime}}(x)\right|$, is non-increasing with the increase of $\lambda$. Mathematically, we have 
Theorem 1: Let $0 \leq \lambda_{1}<\lambda_{2}$ and suppose that $x^{*}\left(\lambda_{1}\right)$ is an optimal solution to the meanabsolute-deviation cost minimization model (4) when $\lambda=\lambda_{1}$ and $x^{*}\left(\lambda_{2}\right)$ is an optimal solution when $\lambda=\lambda_{2}$, then

$$
\sum_{s \in S} p_{s}\left|\xi_{s}\left(x^{*}\left(\lambda_{1}\right)\right)-\sum_{s^{\prime} \in S} p_{s^{\prime}} \xi_{s^{\prime}}\left(x^{*}\left(\lambda_{1}\right)\right)\right| \geq \sum_{s \in S} p_{s}\left|\xi_{s}\left(x^{*}\left(\lambda_{2}\right)\right)-\sum_{s^{\prime} \in S} p_{s^{\prime}} \xi_{s^{\prime}}\left(x^{*}\left(\lambda_{2}\right)\right)\right|
$$

Proof: Since $x^{*}\left(\lambda_{1}\right)$ is an optimal solution when $\lambda=\lambda_{1}$, its objective value is no larger than any other feasible solution. Therefore,

$$
\begin{aligned}
& \sum_{s \in S} p_{s} \xi_{s}\left(x^{*}\left(\lambda_{1}\right)\right)+\lambda_{1} \sum_{s \in S} p_{s}\left|\xi_{s}\left(x^{*}\left(\lambda_{1}\right)\right)-\sum_{s^{\prime} \in S} p_{s^{\prime}} \xi_{s^{\prime}}\left(x^{*}\left(\lambda_{1}\right)\right)\right| \\
& \leq \sum_{s \in S} p_{s} \xi_{s}\left(x^{*}\left(\lambda_{2}\right)\right)+\lambda_{1} \sum_{s \in S} p_{s}\left|\xi_{s}\left(x^{*}\left(\lambda_{2}\right)\right)-\sum_{s^{\prime} \in S} p_{s^{\prime}} \xi_{s^{\prime}}\left(x^{*}\left(\lambda_{2}\right)\right)\right|
\end{aligned}
$$

Similarly, since $x^{*}\left(\lambda_{2}\right)$ is an optimal solution when $\lambda=\lambda_{2}$, it follows that

$$
\begin{aligned}
& \sum_{s \in S} p_{s} \xi_{s}\left(x^{*}\left(\lambda_{2}\right)\right)+\lambda_{2} \sum_{s \in S} p_{s}\left|\xi_{s}\left(x^{*}\left(\lambda_{2}\right)\right)-\sum_{s^{\prime} \in S} p_{s^{\prime}} \xi_{s^{\prime}}\left(x^{*}\left(\lambda_{2}\right)\right)\right| \\
& \leq \sum_{s \in S} p_{s} \xi_{s}\left(x^{*}\left(\lambda_{1}\right)\right)+\lambda_{2} \sum_{s \in S} p_{s}\left|\xi_{s}\left(x^{*}\left(\lambda_{1}\right)\right)-\sum_{s^{\prime} \in S} p_{s^{\prime}} \xi_{s^{\prime}}\left(x^{*}\left(\lambda_{1}\right)\right)\right|
\end{aligned}
$$

Summing up Eqs. (6)-(7), and rearranging terms, we have

$$
\begin{gathered}
\left(\lambda_{1}-\lambda_{2}\right)\left\{\sum_{s \in S} p_{s}\left|\xi_{s}\left(x^{*}\left(\lambda_{1}\right)\right)-\sum_{s^{\prime} \in S} p_{s^{\prime}} \xi_{s^{\prime}}\left(x^{*}\left(\lambda_{1}\right)\right)\right|-\sum_{s \in S} p_{s}\left|\xi_{s}\left(x^{*}\left(\lambda_{2}\right)\right)-\sum_{s^{\prime} \in S} p_{s^{\prime}} \xi_{s^{\prime}}\left(x^{*}\left(\lambda_{2}\right)\right)\right|\right\} \\
\leq 0
\end{gathered}
$$

Since $\lambda_{1}<\lambda_{2}$, Eq. (8) leads to Eq. (5). $\square$

Since parameters $\lambda$ and $p_{s}, s \in S$, are non-negative, the mean-absolute-deviation cost minimization model (4) can be equivalently transformed into the following minimization model by introducing two more non-negative variables $\delta_{s}^{+}$and $\delta_{s}^{-}$for each scenario $s \in S$ :

subject to

$$
\min C_{\mathrm{MAD}}\left(x, \delta_{s}^{+}, \delta_{s}^{-}\right)=\sum_{s \in S} p_{s} \xi_{s}(x)+\lambda \sum_{s \in S} p_{s}\left(\delta_{s}^{+}+\delta_{s}^{-}\right)
$$

$$
\sum_{s^{\prime} \in S} p_{s^{\prime}} \xi_{s^{\prime}}(x)-\xi_{s}(x)=\delta_{s}^{+}-\delta_{s}^{-}, \forall s \in S
$$




$$
\begin{gathered}
\delta_{s}^{+}, \delta_{s}^{-} \geq 0, \forall s \in S \\
x \in X
\end{gathered}
$$

Eq. (10) implies that

$$
\delta_{s}^{+}=\sum_{s^{\prime} \in S} p_{s^{\prime}} \xi_{s^{\prime}}(x)-\xi_{s}(x)+\delta_{s}^{-}
$$

The minimization model (9)-(12) can be rewritten as follows after substituting variables $\delta_{s}^{+}$ with the term on the right-hand side of Eq. (13):

$$
\min _{x \in X} C_{\mathrm{MAD}}\left(x, \delta_{s}^{-}\right)=\sum_{s \in S} p_{s} \xi_{s}(x)+\lambda \sum_{s \in S} p_{s}\left(\sum_{s^{\prime} \in S} p_{s^{\prime}} \xi_{s^{\prime}}(x)-\xi_{s}(x)+2 \delta_{s}^{-}\right)
$$

subject to

$$
\begin{gathered}
\sum_{s^{\prime} \in S} p_{s^{\prime}} \xi_{s^{\prime}}(x)-\xi_{s}(x)+\delta_{s}^{-} \geq 0, \forall s \in S \\
\delta_{s}^{-} \geq 0, \forall s \in S
\end{gathered}
$$

The mean-absolute-deviation cost minimization model has a linear programming form and therefore it is computationally more tractable than the mean-variance cost minimization model. The mean-absolute-deviation cost minimization model has been widely used in revenue management (Lai and Ng, 2005; Lai et al., 2007), stochastic logistics optimization (Yu and Li, 2000; Leung et al., 2002), production planning (Leung et al., 2007a, b) and robust supply chain design (Pan and Nagi, 2010).

There is no doubt that the weighting $\lambda$ has significant impacts on the optimal solution for the mean-absolute-deviation cost minimization model. A wide variability of the weightings has been taken by the researchers for solving different robust optimization problems. For example, Yu and Li (2000) and Leung et al. (2002, 2007a, 2007b) have set the weighting $\lambda=1$; Lai and $\mathrm{Ng}$ (2005) and Lai et al. (2007) have carried out the sensitivity analysis on the weighting $\lambda$ from the value 1 to several tens; Pan and Nagi (2010) gradually increased the value of $\lambda$ from 0 and reported the result when $\lambda$ equals $0,1,10$ and a very large number. These studies generally observe that when the decision-maker is more 
conservative, which corresponds to a larger weighting $\lambda$, the solution obtained by the meanabsolute-deviation cost minimization model incurs a higher expected value of cost. Despite the numerical analysis, these studies have not examined the impact of the weighting in principle. This paper aims to rigorously derive a tight upper bound of the weighting and show that the solution obtained from the mean-absolute-deviation cost minimization model actually may not be an optimal decision provided that value of the weighting is greater than this upper bound.

The remainder of this paper is organized as follows. Section 2 first uses a straightforward example to illustrate the difference between the mean-absolute-deviation model and multiobjective minimization model and impact of the weighting. It also defines the absolute domination relation. Section 3 gives a tight upper bound of the weighting. Section 4 further analyzes the impact of the weighting $\lambda$ using numerical examples. Conclusions are presented in Section 5.

2. Definition of the absolute domination and differences between the mean-absolutedeviation cost minimization model and multiobjective minimization model

From pure mathematical point of view, the mean-absolute-deviation cost minimization model is a weighted transformation of the bi-objective minimization model aiming to simultaneously minimize the mean and absolute deviation of the random cost:

$$
\min _{x \in X}\left(\begin{array}{l}
C(x) \\
A D(x)
\end{array}\right)=\left(\begin{array}{l}
\sum_{s \in S} p_{s} \xi_{s}(x) \\
\sum_{s \in S} p_{s}\left|\xi_{s}(x)-\sum_{s^{\prime} \in S} p_{s^{\prime}} \xi_{s^{\prime}}(x)\right|
\end{array}\right)
$$

However, such an interpretation is inappropriate to a certain extent as demonstrated by a hypothetical example as follows: 
Illustrative Example: Suppose that a company can lease machines at the beginning of a year to produce products over the year. It is assumed that leasing price per machine is 500 USD and each machine can produce a maximum of 1000 units of the products. Marketing department of the company predicts that there are two possible demand scenarios $s_{1}$ and $s_{2}$ for the products in the year: $d_{s_{1}}=1200$ units and $d_{s_{2}}=1500$ units, namely, $S=\left\{s_{1}, s_{2}\right\}$. The probability of each demand scenario happening is $p_{s_{1}}=\varepsilon$ and $p_{s_{2}}=1-\varepsilon$, where parameter $\varepsilon \in(0,1)$. The demand must be fulfilled. If the production capacity of the leased machines is not enough to meet the demand, the company will purchase the lacking products from other companies at the price of $1 \mathrm{USD} / \mathrm{unit}$. This company is risk averse and aims to find the optimal number of machines to lease by taking into account two factors: minimization of the expected cost and the relevant absolute deviation.

There are two potentially optimal solutions for the company to meet the demand: lease one or two machines, namely, $X=\{1,2\}$. Let solutions $x_{1}=1$ and $x_{2}=2$. The incurred cost for each given demand scenario with respect to a feasible solution can be calculated as follows:

$$
\begin{gathered}
\xi_{s_{1}}\left(x_{1}\right)=x_{1} \times 500 \mathrm{USD} / \text { machine }+\left(d_{s_{1}}-1000\right) \times 1 \mathrm{USD} / \text { unit }=700 \mathrm{USD} \\
\xi_{s_{1}}\left(x_{2}\right)=x_{2} \times 500 \mathrm{USD} / \text { machine }=1000 \mathrm{USD} \\
\xi_{s_{2}}\left(x_{1}\right)=x_{1} \times 500 \mathrm{USD} / \text { machine }+\left(d_{s_{2}}-1000\right) \times 1 \mathrm{USD} / \text { unit }=1000 \mathrm{USD} \\
\xi_{s_{2}}\left(x_{2}\right)=x_{2} \times 500 \mathrm{USD} / \text { machine }=1000 \mathrm{USD}
\end{gathered}
$$

The expected cost and absolute deviation of cost for each feasible solution can be hence calculated by

$$
C\left(x_{1}\right)=\sum_{s \in S} p_{s} \xi_{s}\left(x_{1}\right)=\varepsilon \times 700+(1-\varepsilon) \times 1000=1000-300 \varepsilon
$$




$$
\begin{gathered}
A D\left(x_{1}\right)=\sum_{s \in S} p_{s}\left|\xi_{s}\left(x_{1}\right)-C\left(x_{1}\right)\right|=600 \varepsilon(1-\varepsilon) \\
C\left(x_{2}\right)=\sum_{s \in S} p_{s} \xi_{s}\left(x_{2}\right)=\varepsilon \times 1000+(1-\varepsilon) \times 1000=1000 \\
A D\left(x_{2}\right)=\sum_{s \in S} p_{s}\left|\xi_{s}\left(x_{2}\right)-C\left(x_{2}\right)\right|=0
\end{gathered}
$$

According to Eqs. (22)-(25), it can be concluded that that both solutions $x_{1}$ and $x_{2}$ are Pareto optimal to the bi-objective minimization model (17) according to the multiobjective optimality conditions (Chankong and Haimes, 1983). This is because the following two vectors are non-dominated:

$$
\begin{gathered}
\left(\begin{array}{l}
C\left(x_{1}\right) \\
A D\left(x_{1}\right)
\end{array}\right)=\left(\begin{array}{l}
1000-300 \varepsilon \\
600 \varepsilon(1-\varepsilon)
\end{array}\right) \\
\left(\begin{array}{l}
C\left(x_{2}\right) \\
A D\left(x_{2}\right)
\end{array}\right)=\left(\begin{array}{l}
1000 \\
0
\end{array}\right)
\end{gathered}
$$

In other words, there is no difference between these two solutions and whether $x_{1}$ or $x_{2}$ is chosen depends on the attitude toward risk in the mean-absolute-deviation cost minimization model. As a matter of fact, solution $x_{1}$ is always preferable to solution $x_{2}$ regardless of the attitude toward risk because in scenario $s_{1}$, solution $x_{1}$ is better than solution $x_{2}$ in that $x_{1}$ has lower cost and in scenario $s_{2}$, both solutions $x_{1}$ and $x_{2}$ have the same cost. Therefore, the company should definitely choose solution $x_{1}$. The drawback of the mean-absolutedeviation cost minimization model illustrated by this example is due to the inherent difference between the mean-absolute-deviation cost minimization and multiobjective optimization problems in the criterion to evaluate a solution. A mean-absolute-deviation cost minimization problem should take into account the cost incurred for each possible scenario rather than the expected cost and absolute deviation of cost. To deal with this issue, we define the following absolute domination as an extension of the conventional domination used in the multiobjective optimization studies: 
Definition: A solution $x \in X$ absolutely dominates solution $y \in X$ if and only if $\xi_{s}(x) \leq \xi_{s}(y), \forall s \in \Omega$, and there exists a particular scenario $\bar{s} \in \Omega$ satisfying $\xi_{\bar{s}}(x)<\xi_{\bar{s}}(y)$. Solution $y \in X$ is absolutely dominated by solution $x \in X$ provided that solution $x$ absolutely dominates solution $y$. solution $y \in X$ is absolutely non-dominated if $y$ is not absolutely dominated by any solution $x \in X$.

According to this definition, solution $x_{2}$ is absolutely dominated by solution $x_{1}$ for the illustrative example.

We now use the illustrative example to analyze impact of the weighting on the solution of the mean-absolute-deviation cost minimization model. The sum of the expected cost and the absolute deviation of cost in Eq. (4) for each feasible solution of the illustrative example can be calculated as follows:

$$
\begin{gathered}
C_{\mathrm{MAD}}\left(x_{1}\right)=C\left(x_{1}\right)+\lambda A D\left(x_{1}\right)=1000-300 \varepsilon+600 \lambda \varepsilon(1-\varepsilon) \\
C_{\mathrm{MAD}}\left(x_{2}\right)=C\left(x_{2}\right)+\lambda A D\left(x_{2}\right)=1000
\end{gathered}
$$

If we set the weighting $\lambda=0.5$ in the mean-absolute-deviation model (4), it follows that

$$
C_{\mathrm{MAD}}\left(x_{1}\right)=1000-300 \varepsilon^{2}<C_{\mathrm{MAD}}\left(x_{2}\right)=1000
$$

Eq. (30) implies that the absolutely non-dominated solution $x_{1}$ is the solution to the model. However, when the weighting $\lambda=1.0$ and $\varepsilon \in(0,0.5)$, we have

$$
C_{\mathrm{MAD}}\left(x_{1}\right)=1000+300 \varepsilon(1-2 \varepsilon)>C_{\mathrm{MAD}}\left(x_{2}\right)=1000
$$

Eq. (31) indicates that the worse solution $x_{2}$ (since it is absolutely dominated by $x_{1}$ ) is chosen by the mean-absolute-deviation cost minimization model. The illustrative example clearly demonstrates the importance of the weighting for the mean-absolute-deviation cost minimization model. It is thus necessary to derive a range of the weighting such that the 
optimal solution of the mean-absolute-deviation cost minimization model excludes all absolutely dominated solutions.

\section{A tight upper bound for the weighting $\lambda$}

The illustrative example shows that the mean-absolute-deviation cost minimization model is inherently different from the multiobjective minimization model. It is possible to obtain an absolutely dominated solution, namely, a worse solution by the mean-absolutedeviation cost minimization model for some value of the weighting $\lambda$. We now rigorously prove that none of the absolutely dominated solutions will be an optimal solution to the mean-absolute-deviation cost minimization model for the weighting $\lambda \in(0,0.5]$.

It is straightforward to prove the lemma as follows:

Lemma: For any two real numbers $a, b \in \mathfrak{R}$, we have

$$
\begin{gathered}
|a|-|b| \leq|a-b| \\
|a+b|<|a|+|b|, \text { if } a b<0
\end{gathered}
$$

Based on the lemma above, we have the following interesting theorem:

Theorem 2: If $x^{*}$ is an optimal solution to the mean-absolute-deviation cost minimization model (4) with a specific weighting $\lambda \in(0,0.5]$, then $x^{*}$ is an absolutely non-dominated solution.

Proof: Suppose that the optimal solution $x^{*}$ is not an absolutely non-dominated solution. Hence, there is a solution $y \in X$ absolutely dominating the optimal solution $x^{*}$, namely:

$$
\xi_{s}(y) \leq \xi_{s}\left(x^{*}\right), \forall s \in S
$$

and there exists at least one particular scenario $\bar{s} \in S$ such that

$$
\xi_{\bar{s}}(y)<\xi_{\bar{s}}\left(x^{*}\right)
$$


Therefore the average cost defined by Eq. (17) with respect to solutions $x^{*}$ and $y$ satisfies:

$$
C(y)<C\left(x^{*}\right)
$$

According to the lemma and Eqs. (34), (35) and (36), we have

$$
\begin{aligned}
& \sum_{s \in S} p_{s}\left[\left|\xi_{s}(y)-C(y)\right|-\left|\xi_{s}\left(x^{*}\right)-C\left(x^{*}\right)\right|\right] \\
& \leq \sum_{s \in S} p_{s}\left|\left[\xi_{s}(y)-\xi_{s}\left(x^{*}\right)\right]+\left[C\left(x^{*}\right)-C(y)\right]\right| \\
& =\left\{\sum_{s \in S \backslash \bar{s}} p_{s}\left|\left[\xi_{s}(y)-\xi_{s}\left(x^{*}\right)\right]+\left[C\left(x^{*}\right)-C(y)\right]\right|\right\}+p_{\bar{s}}\left|\left[\xi_{\bar{s}}(y)-\xi_{\bar{s}}\left(x^{*}\right)\right]+\left[C\left(x^{*}\right)-C(y)\right]\right| \\
& <\left\{\sum_{s \in S \backslash \bar{s}} p_{s}\left[\left|\xi_{s}(y)-\xi_{s}\left(x^{*}\right)\right|+\left|C\left(x^{*}\right)-C(y)\right|\right]\right\}+p_{\bar{s}}\left|\xi_{\bar{s}}(y)-\xi_{\bar{s}}\left(x^{*}\right)\right|+p_{\bar{s}}\left|C\left(x^{*}\right)-C(y)\right| \\
& =\left\{\sum_{s \in S \backslash \bar{s}} p_{s}\left[\xi_{s}\left(x^{*}\right)-\xi_{s}(y)+C\left(x^{*}\right)-C(y)\right]\right\}+p_{\bar{s}}\left[\xi_{\bar{s}}\left(x^{*}\right)-\xi_{\bar{s}}(y)\right]+p_{\bar{s}}\left[C\left(x^{*}\right)-C(y)\right] \\
& =\sum_{s \in S} p_{s} \xi_{s}\left(x^{*}\right)-\sum_{s \in S} p_{s} \xi_{s}(y)+C\left(x^{*}\right)-C(y) \\
& =2\left[C\left(x^{*}\right)-C(y)\right]
\end{aligned}
$$

For any $\lambda \in(0,0.5]$, it can thus be seen that

$$
\begin{aligned}
& C_{\mathrm{MAD}}(y)-C_{\mathrm{MAD}}\left(x^{*}\right) \\
& =C(y)+\lambda \sum_{s \in S} p_{s}\left|\xi_{s}(y)-C(y)\right|-\left[C\left(x^{*}\right)+\lambda \sum_{s \in \Omega} p_{s}\left|\xi_{s}\left(x^{*}\right)-C\left(x^{*}\right)\right|\right] \\
& =\left[C(y)-C\left(x^{*}\right)\right]+\lambda \sum_{s \in S} p_{s}\left[\left|\xi_{s}(y)-C(y)\right|-\left|\xi_{s}\left(x^{*}\right)-C\left(x^{*}\right)\right|\right] \\
& <\left[C(y)-C\left(x^{*}\right)\right]+2 \lambda\left[C\left(x^{*}\right)-C(y)\right] \\
& =(2 \lambda-1)\left[C\left(x^{*}\right)-C(y)\right] \leq 0
\end{aligned}
$$

In other words, we have

$$
C_{\mathrm{MAD}}(y)<C_{\mathrm{MAD}}\left(x^{*}\right)
$$

Eq. (39) implies a contradiction that $x^{*}$ is the optimal solution to the mean-absolute-deviation cost minimization model (4).

Theorem 2 shows that 0.5 is an upper bound on the weighting $\lambda$ to guarantee that none of the absolutely dominated solutions is an optimal solution to the mean-absolute-deviation cost minimization model. We now demonstrate the tightness of this bound using the illustrative 
example. For the illustrative example, let us take $\lambda=0.5+\delta$ where $\delta>0$. According to Eqs. (28)-(29), it can be seen that

$$
C_{\mathrm{MAD}}\left(x_{2}\right)-C_{\mathrm{MAD}}\left(x_{1}\right)=300 \varepsilon \times[(1+2 \delta) \varepsilon-2 \delta]
$$

We can thus choose an $\varepsilon<2 \delta /(1+2 \delta)$ and hence $C_{\mathrm{MAD}}\left(x_{2}\right)<C_{\mathrm{MAD}}\left(x_{1}\right)$. Namely, solution $x_{2}$, which is absolutely dominated by solution $x_{1}$, is yielded by the mean-absolute-deviation cost minimization model.

We note that given a specific problem, $\lambda>0.5$ does not necessarily mean that an absolutely dominated solution will be obtained from the mean-absolute-deviation cost minimization model. Moreover, for some practical problems, it might be possible that the decision maker is so risk averse that she chooses the value of $\lambda$ larger than 0.5 . Therefore it is necessary to check whether the optimal solution to the mean-absolute deviation cost minimization model, denoted by $x^{*}$, is absolutely dominated. This can be implemented by solving the minimization model below:

[Check Model]

$$
\min _{x \in X} C=\sum_{s \in \Omega} p_{s} \xi_{s}(x)
$$

subject to

$$
\xi_{s}(x) \leq \xi_{s}\left(x^{*}\right), \forall s \in S
$$

Theorem 3: If the optimal objective function value of (41) equals that of the mean-absolutedeviation cost minimization model (4), then $x^{*}$ is absolutely non-dominated. Otherwise $x^{*}$ is absolutely dominated.

The proof of Theorem 3 is straightforward. Denote by $\hat{x}$ the optimal solution to Eqs. (41)(42). If $\sum_{s \in \Omega} p_{s} \xi_{s}(\hat{x})=\sum_{s \in \Omega} p_{s} \xi_{s}\left(x^{*}\right)$, namely, $x^{*}$ is absolutely non-dominated, then we have $\xi_{s}(\hat{x})=\xi_{s}\left(x^{*}\right), \forall s \in S$ as a consequence of the constraints (42). Therefore, either $\hat{x}=x^{*}$ or $\hat{x}$ and $x^{*}$ lead to the same cost in any scenario $s \in S$. In other words, there is no difference between $\hat{x}$ and $x^{*}$ whatever criterion is being used. If $x^{*}$ is absolutely dominated by $\hat{x}, \hat{x}$ is 
absolutely non-dominated. In such a circumstance, we can either decrease the value of $\lambda$ and reoptimize the mean-absolute-deviation cost minimization model (4), or use $\hat{x}$ as the final decision in place of $x^{*}$.

\section{Numerical examples}

In this section we use numerical examples to test whether it is often the case that the solution of the mean-absolute-deviation cost minimization model is absolutely dominated when $\lambda>0.5$. We had better test the solutions obtained by existing studies (Yu and Li, 2000; Leung et al., 2002, 2007a, b, Lai and Ng, 2005; Lai et al., 2007, Pan and Nagi, 2010) using the model (41)-(42). Nevertheless, the data used in these studies are not readily available. Therefore, we have to use other numerical examples.

To ensure that the scenarios and their probabilities are representative, we use several portfolio optimization examples based on historical data of the S\&P 100. The S\&P 100 is comprised of 100 leading U.S. stocks with exchange-listed options. Constituents of the S\&P 100 are selected for sector balance and represent almost $45 \%$ of the market capitalization of the U.S. equity markets. The stocks in the S\&P 100 are generally among the largest and most established companies. We download the S\&P 100 historical data from Option Trading Tips (2011). Since this dataset is incomplete in that the trading information for some stocks on some days is missing, we choose 91 stocks and analyze the monthly rate of return from January 2000 to December 2009 for each stock. Each month is considered as a scenario, and altogether there are 120 scenarios. We generate 15 test instances, each of which having 12 or 24 scenarios (months), as shown in the first three columns of Table 1.

To formulate the problem, let $R_{j}$ be a random variable representing the rate of return per period of stock $j, j=1,2 \cdots n, n=91$. Denote by $r_{j}$ the expected value of $R_{j}$, and $r_{j t}$ the rate of return of stock $j$ in scenario $t, t=1,2 \cdots T . T$ is equal to 12 or 24 in the test instances. 
Without loss of generality, we assume that the total fund for investment is 1 . Let $u_{j}$ be the maximum money that can be invested in stock $j$ and let $x_{j}$ be the money that will be invested in stock $j$. We set $u_{j}=0.2$ for all $j$. The optimal portfolio selection problem can be formulated as the mean-absolute-deviation model with the weighting $\lambda$ :

$$
\begin{aligned}
\min _{x_{j}} C_{\mathrm{MAD}} & =-\sum_{j=1}^{n} r_{j} x_{j}+\lambda \sum_{j=1}^{n} \frac{1}{T} \sum_{t=1}^{T}\left|r_{j t} x_{j}-r_{j} x_{j}\right| \\
& =\sum_{j=1}^{n}\left(\lambda \frac{1}{T} \sum_{t=1}^{T}\left|r_{j t}-r_{j}\right|-r_{j}\right) x_{j}
\end{aligned}
$$

subject to

$$
\begin{gathered}
\sum_{j=1}^{n} x_{j}=1 \\
0 \leq x_{j} \leq u_{j}, j=1,2 \cdots n
\end{gathered}
$$

We solve the above mean-absolute-deviation model for the 15 test instances with parameter $\lambda \in\{1,2,5,10,100\}$, and then use the minimization model expressed by Eqs. (41)(42) to check whether the optimal solution is absolutely dominated by another solution. The results are shown in Table 1, where "Y" represents that the obtained solution is absolutely dominated by another solution. According to Table 1 , a larger $\lambda$ tends to yield an absolutely dominated solution. In all the instances whenever a $\lambda$ yields an absolutely dominated solution, all larger $\lambda$ will also yield absolutely dominated solutions. The results in Table 1 demonstrate that the solutions obtained by the mean-absolute-deviation model with 12 or 24 scenarios are frequently absolutely dominated. It should be mentioned that the larger the number of scenarios is, the less likely that a solution is to be absolutely dominated. This is because if a solution is absolutely dominated, there must be another solution that is not worse than it in every scenario. In practice, planners in industries such as logistics and production generally can only propose a few scenarios due to the insufficiency of historical data, for example, 4 scenarios for one example and 1 to 12 scenarios for the other example in $\mathrm{Yu}$ and 
Li (2000), 4 scenarios in Leung et al. (2002, 2007a, 2007b), 4 scenarios in Lai and $\mathrm{Ng}$ (2005) and 3 scenarios in Lai et al. (2007). Therefore, the solutions obtained by these studies are very likely to be absolutely dominated. This highlights the importance of our study.

Table 1 Absolute domination results

\begin{tabular}{cccccccc}
\hline ID & Year & \# Month & $\lambda=1$ & $\lambda=2$ & $\lambda=5$ & $\lambda=10$ & $\lambda=100$ \\
\hline 1 & 2000 & 12 & & $\mathrm{Y}$ & $\mathrm{Y}$ & $\mathrm{Y}$ & $\mathrm{Y}$ \\
2 & 2001 & 12 & $\mathrm{Y}$ & $\mathrm{Y}$ & $\mathrm{Y}$ & $\mathrm{Y}$ & $\mathrm{Y}$ \\
3 & 2002 & 12 & $\mathrm{Y}$ & $\mathrm{Y}$ & $\mathrm{Y}$ & $\mathrm{Y}$ & $\mathrm{Y}$ \\
4 & 2003 & 12 & & $\mathrm{Y}$ & $\mathrm{Y}$ & $\mathrm{Y}$ & $\mathrm{Y}$ \\
5 & 2004 & 12 & $\mathrm{Y}$ & $\mathrm{Y}$ & $\mathrm{Y}$ & $\mathrm{Y}$ & $\mathrm{Y}$ \\
6 & 2005 & 12 & & $\mathrm{Y}$ & $\mathrm{Y}$ & $\mathrm{Y}$ & $\mathrm{Y}$ \\
7 & 2006 & 12 & $\mathrm{Y}$ & $\mathrm{Y}$ & $\mathrm{Y}$ & $\mathrm{Y}$ & $\mathrm{Y}$ \\
8 & 2007 & 12 & & $\mathrm{Y}$ & $\mathrm{Y}$ & $\mathrm{Y}$ & $\mathrm{Y}$ \\
9 & 2008 & 12 & & & & $\mathrm{Y}$ & $\mathrm{Y}$ \\
10 & 2009 & 12 & & $\mathrm{Y}$ & $\mathrm{Y}$ & $\mathrm{Y}$ & $\mathrm{Y}$ \\
11 & $2000-2001$ & 24 & & $\mathrm{Y}$ & $\mathrm{Y}$ & $\mathrm{Y}$ & $\mathrm{Y}$ \\
12 & $2002-2003$ & 24 & $\mathrm{Y}$ & $\mathrm{Y}$ & $\mathrm{Y}$ & $\mathrm{Y}$ & $\mathrm{Y}$ \\
13 & $2004-2005$ & 24 & & $\mathrm{Y}$ & $\mathrm{Y}$ & $\mathrm{Y}$ & $\mathrm{Y}$ \\
14 & $2006-2007$ & 24 & & & $\mathrm{Y}$ & $\mathrm{Y}$ & $\mathrm{Y}$ \\
15 & $2008-2009$ & 24 & & & & & \\
\hline
\end{tabular}

\section{Conclusions}

This paper has analyzed the significant impact of the weighting involved in the meanabsolute-deviation cost minimization model and pointed out the differences between the mean-absolute-deviation cost minimization model and the multiobjective optimization problems. These differences enable us to define the absolute domination relation. The meanabsolute-deviation cost minimization model, despite its robustness, may yield an absolutely dominated solution. The tight upper bound on the weighting for the absolute deviation of cost is derived, whereby all the absolutely dominated solutions are excluded from the mean- 
absolute-deviation cost minimization model. We also presented a model for checking whether an absolutely dominated solution is obtained in problems with the weighting larger than the tight upper bound. Numerical experiments demonstrate that the solutions obtained by the mean-absolute-deviation model with 12 or 24 scenarios are frequently absolutely dominated. Hence, our analysis on the weighting has practical implications for planners in industries such as logistics and production where in general only a small number of scenarios can be considered.

\section{Acknowledgements}

This study is supported by the research grants -WBS No. R-264-000-244-720 and WBS No. R-302-000-014-720- from the NOL Fellowship Programme of Singapore.

\section{References}

Ben-Tal A, El Ghaoui L and Nemirovski A (2009). Robust Optimization, Princeton University Press.

Chankong V and Haimes Y Y (1983). Multiobjective decision making: theory and methodology. North Holland, New York.

Konno $\mathrm{H}$ and Yamazaki H (1991). Mean-absolute deviation portfolio optimization model and its applications to Tokyo stock market. Manage Sci 37(5), 519-531.

Lai K-K and Ng W-L (2005). A stochastic approach to hotel revenue optimization. Comput Oper Res 32(5), 1059-1072.

Lai K K, Wang M, Liang L (2007). A stochastic approach to professional services firms' revenue optimization. Eur J Oper Res 182(3), 971-982. 
Leung S C H, Wu Y, Lai K K (2002). A robust optimization model for a cross-border logistics problem with fleet composition in an uncertain environment. Math Comput Model 36(11-13), 1221-1234.

Leung S C H, Lai K K, Ng W-L, Wu Y (2007a). A robust optimization model for production planning of perishable products. J Oper Res Soc 58(4), 413-422.

Leung S C H, Tsang S O S, Ng W L, Wu Y (2007b). A robust optimization model for multisite production planning problem in an uncertain environment. Eur J Oper Res 181(1), 224-238.

Mansini R, Ogryczak W, Speranza M G (2003). LP solvable models for portfolio optimization: a classification and computational comparison. IMA J Manage Math 14, 187-220.

Markowitz H M (1952). Portfolio selection. J Financ 7, 77-91.

Mulvey J M, Vanderbei R J, Zenios S A (1995). Robust optimization of large-scale systems. Oper Res 43(2), 264-281.

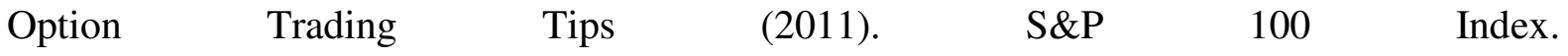
http://www.optiontradingtips.com/resources/historical-data/sp100.html. Accessed May 4, 2011.

Pan F, Nagi R (2010). Robust supply chain design under uncertain demand in agile manufacturing. Comput Oper Res 37(4), 668-683.

Yu C-S, Li H-L (2000). A robust optimization model for stochastic logistic problems. Int J Prod Econ 64(1-3), 385-397. 